Pacific Journal of Mathematics

GENERATORS FOR TWO GROUPS RELATED TO THE BRAID 


\title{
GENERATORS FOR TWO GROUPS RELATED TO THE BRAID GROUP
}

\author{
Hugh M. HILDEN
}

Let $D$ be the unit ball in 3-space and let $A_{k}$ be a set of $k$ proper disjoint arcs in $D$ lying in the $x-z$ plane. The group, $\mathscr{K}_{2 k}$, of orientation preserving homeomorphisms of boundary $D$ leaving the set $A_{k} \cap$ boundary $D$ invariant, modulo those isotopic to the identity via an isotopy fixing the set $A_{k} \cap$ boundary $D$, is a natural homomorphic image of the $2 k$ string braid group of the sphere via a homomorphism with kernel $Z_{2}$.

In this paper, finite sets of generators are explicitly determined for the subgroups of $\mathscr{K}_{2 k}$ generated by and

(1) homeomorphisms of $D$ leaving the set $A_{k}$ invariant,

(2) homeomorphisms of $D$ leaving the set $A_{k}$ fixed pointwise.

1. Introduction. Let $D$ be the unit ball in 3-space and let $P$ be a set of $n$ points on the boundary of $D$ (denoted $\partial D$ ). The group of orientation perserving homeomorphisms of $\partial D$ leaving $P$ invariant, modulo the subgroup of those isotopic to the identity via an isotopy fixing $P$, will be denoted $\mathscr{C}_{n}$. The group $\mathscr{C}_{n}$ is a subgroup of index two of the full mapping class group of the sphere with $n$ points removed. (The full group contains equivalence classes of orientation reversing homeomorphisms.) There is a natural map of the $n$-string braid group of the sphere onto $\mathscr{C}_{n}$ whose kernel is $Z_{2}$. For a definition of the braid group, the mapping class group of a surface, their relationship and for a description of a presentation of the braid group the reader is referred to Joan Birman's work ([1] and [2]).

Let $A$ be a set of $k$ disjoint proper arcs in $D$ lying in the $x-z$ plane. Suppose that $P$ is the set of $2 k$ points $A \cap \partial D$. Let $\mathscr{A}_{k}$ be the subgroup of $\mathscr{A}_{2 l}$ generated by equivalence classes of homeomorphisms of $D$ leaving the set $A$ invariant, restricted to $\partial D$. Let $\mathscr{F}_{k}$ be the subgroup of $\mathscr{A}_{k}$ generated by homeomorphisms leaving the set $A$ fixed pointwise. Naturally the groups $\mathscr{A}_{k}$ and $\mathscr{F}_{k}$ will depend on the choice of the set $A$. However, given any two such sets $A$ and $A^{\prime}$, there is an orientation preserving homeomorphism of $D$ taking $A$ onto $A^{\prime}$, so the groups $\mathscr{A}_{k}$ and $\mathscr{A}_{k}^{\prime} ; \mathscr{F}_{k}$ and $\mathscr{F}_{k}^{\prime}$ will be isomorphic. The purpose of this paper is to determine, explicitly, a set of generators for the group $\mathscr{A}_{k}$ and $\mathscr{F}_{k}$ for one particular choice of the set $A$.

The groups $\mathscr{A}_{k}$ and $\mathscr{F}_{k}$ arise in knot theory. For example, 
suppose we have two knots $\left(S^{3}, k\right)$ and $\left(S^{3}, k^{\prime}\right)$ represented as closed braids. Let us consider the question, "When is there an orientation preserving homeomorphism of $S^{3}$ taking $k$ onto $k^{\prime}$ ?" (i.e. When are the knots the same?) Let $A_{1}$ equal $D$ intersected with a set of $k$ concentric circles lying in the $x-z$ plane centered at $(1,0,0)$ and having radii between $1 / 2$ and $11 / 2$. Let $\left(D^{\prime}, A_{1}^{\prime}\right)$ be another copy of $(D, A)$ and let $i$ be the identification map of $D$ with $D^{\prime}$, restricted to $\partial D$. Since $\left(S^{3}, k\right)$ and $\left(S^{3}, k^{\prime}\right)$ are represented as closed braids, we can explicitly construct homeomorphisms $\varphi$ and $\psi$ of $\partial D$ fixed on $\partial D \cap$ $\{z \geqq 0\}$ such that

$$
\left(S^{3}, k\right)=\left(D, A_{1}\right) \bigcup_{i \varphi}\left(D^{\prime}, A_{1}^{\prime}\right) \text { and }\left(S^{3}, k^{\prime}\right)=\left(D, A_{1}\right) \bigcup_{i \psi}\left(D^{\prime}, A_{1}^{\prime}\right) .
$$

The sewing homeomorphisms $\varphi$ and $\psi$ can be considered both as elements of the $k$-string braid group $\mathscr{C}_{k}$ of the disc $\partial D \cap\{z<0\}$ and of $\mathscr{C}_{2 k}$. (Unlike the case of the sphere, the group $\mathscr{C}_{k}$, defined in a manner analogous to $\mathscr{N}_{k}$, is isomorphic to the $k$-string braid group, so we identify the two.) The conjugacy problem has been solved in the group $\mathscr{C}_{k}$ (see [4]) and it is not hard to see that if $[\varphi]$ is conjugate to $[\psi]$, then $\left(S^{3}, k\right) \equiv\left(S^{3}, k^{\prime}\right)$. However, there are examples of the type $\left(D, A_{1}\right) \bigcup_{i \alpha}\left(D^{\prime}, A_{1}^{\prime}\right) \equiv\left(D, A_{1}\right) \bigcup_{i \beta}\left(D^{\prime}, A_{1}^{\prime}\right)$ with $\alpha$ and $\beta$ not conjugate, (this can be done with three string, two crossing representations of the trivial knot) so it is reasonable to investigate another equivalence relation on braids with larger equivalence classes. Suppose $[\alpha] \equiv[\beta]$ in $\mathscr{A}_{2 k}$ if they belong to the same double coset $\mathscr{A}_{k} \alpha \mathscr{A}_{k}$ of $\mathscr{A}_{2 k}$. If $[\varphi] \equiv[\psi]$, one can explicitly construct a homeomorphism of $\left(S^{3}, k\right)=\left(D, A_{1}^{\prime}\right) \bigcup_{i \varphi}\left(D^{\prime}, A_{1}^{\prime}\right)$ onto $\left(S^{3}, k^{\prime}\right)=$ $\left(D, A_{1}\right) \bigcup_{i \varphi}\left(D^{\prime}, A_{1}^{\prime}\right)$. The equivalence relation "三” is somewhat analogous to the definition of equivalence of Heegaard splittings of a three manifold.

A solution of the double coset problem (Given $[\alpha]$ and $[\beta] \in \mathscr{C}_{2 k}$ when does $[\alpha] \in \mathscr{A}_{k}[\beta] \mathscr{A}_{k}$ ?) might shed some light on the knot problem.

The organization of this paper is as follows: In $\S 2$ we develop notation, define a set of elements of $\mathscr{A}_{k}$, and prove some technical lemmas. In $\S 3$ we state and prove the main theorem of the paper. In $\S 4$ we give generators for the groups $\mathscr{A}_{k}$ and $\mathscr{F}_{k}$. In $\S 5$ we make concluding remarks relating the groups $\mathscr{A}_{k}$ and $\mathscr{F}_{k}$ to other problems in mathematics.

Throughout the paper we shall work in the P. L. category and all curves, homeomorphisms, isotopies, etc., will be assumed piecewise linear without it being explicitly stated. We shall assume the reader knows much of what is in [3] and we shall refer him there for definitions of such terms as isotopy, ambient isotopy, regular neigh- 
borhood, and locally unknotted.

2. Definition of the generators. In this section a set of elements of $\mathscr{A}_{n}$ is defined which will ultimately be shown to generate $\mathscr{A}_{n}$. We begin by defining certain subsets of the ball $D$ where $D$ is represented in 3 -space as $\{(x, y, z) \mid x \leqq 0\} \cup\{\infty\}$. In the sequel we shall define sets in the manner $S=\{x, y, z$ satisfying certain conditions $\}$ rather than $\{(x, y, z) \mid x, y, z$ satisfies certain conditions $\}$. For example $D=\{x \leqq 0\} \cup\{\infty\}$. Whenever a set of objects is indexed by $i$, we shall assume $1 \leqq i \leqq n$ unless explicitly stated otherwise. At this point the reader should refer to Figure 1.
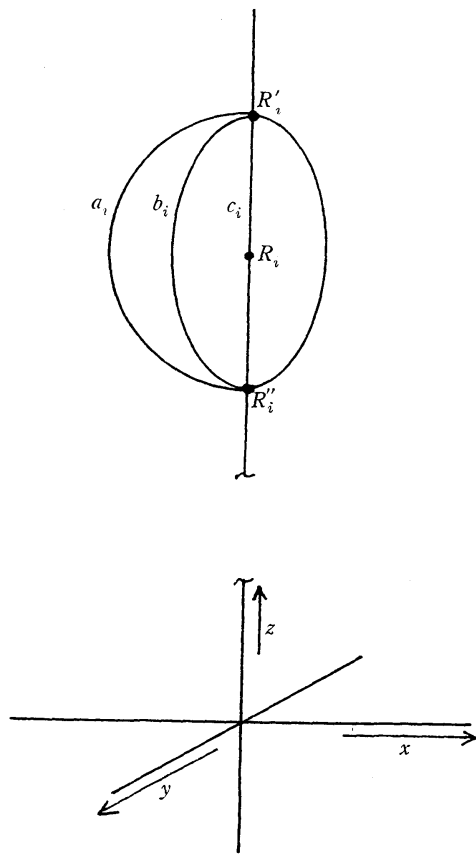

Figure 1

Let $P_{z y}, P_{z x}$, and $Q$ (for quarter space) be the set $\{x=0\},\{y=0$, $x \leqq 0\}$, and $\{x \leqq 0, y \geqq 0\}$ respectively. Let $R_{i}, R_{i}^{\prime}$, and $R_{i}^{\prime \prime}$ be the points $(0,0,8 i),(0,0,8 i+2)$, and $(0,0,8 i-2)$ respectively. Let $c_{i}$ be the line segment $\left[R_{i}^{\prime}, R_{i}^{\prime \prime}\right]$ and let $a_{i}$ and $b_{i}$ be the semicircles with diameter $c_{i}$ contained in the half planes $P_{z x}$ and $P_{z y} \cap Q$ resrectively. Let $A=\mathrm{U}_{i} a_{i}, B=\mathrm{U}_{i} b_{i}$ and $C=\bigcup_{i} c_{i}$. Let $H_{i}$ and $K_{i}$ be the hemisphere, and half ball $\left\{\left\|P-R_{i}\right\|=2\right\}$ and $\left\{\left\|P-R_{i}\right\| \leqq 2\right\}$ respectively, where \|\| is the usual 3-space distance. Note that $a_{i}$ and $b_{i}$ are contained in $H_{i}$. Finally, let $F_{i}=H_{i} \cap Q$ and $E_{i}=K_{i} \cap P_{z x}$, note that $\partial F_{i}=a_{i} \cup b_{i}$ and $\partial E_{i}=a_{i} \cup c_{i}$, and define $F=\bigcup_{i} F_{i}$ and $E=\bigcup_{i} E_{i}$. 
Next we shall define a set of isotopies. In what follows a point moving on a circle in the plane $P_{z y}$ will be said to be moving positively if it appears to be moving counterclockwise to an observer in the right half space $\{x>0\}$. An isotopy $\theta_{t}$ is isometric if $\left\|\theta_{t}(P)-\theta_{t}(Q)\right\|=$ $\|P-Q\|$ for all $P$ and $Q$ in the domain of definition of $\theta_{t}$. An isotopy $\theta_{t}$ will be called a parallel isotopy if the vector $\overrightarrow{\theta_{t}(P) \theta_{t}(Q)}$ is parallel to the vector $\overrightarrow{P Q}$ for all $P$ and $Q$ in the domain of definition of $\theta_{t}$.

1. $\tau_{i}$. Let $\tau_{i}(t)$ (the flip of the $i^{\text {th }}$ arc) be the isometric isotopy of the inclusion map $K_{i} \rightarrow D$ that rotates $K_{i}$ through an angle of $180^{\circ}$ about an axis perpendicular to $P_{y z}$ through the point $R_{\imath}$. We insist that the point $\tau_{i}(t)\left(R_{\imath}^{\prime}\right)$ move positively and with constant speed.

2. $\sigma_{i}, 1 \leqq i \leqq n-1$. Let $\sigma_{i}(t)$ (the exchange of the $i^{\text {th }}$ and $i+1^{\text {st }}$ arcs) be the isometric isotopy of the inclusion map $K_{i} \cup K_{i+1} \rightarrow D$ that rotates $K_{i}$ and $K_{i+1}$ through an angle of $180^{\circ}$ about an axis perpendicular to $P_{z y}$ and through the midpoint of $\left[R_{i}, R_{i+1}\right]$. We insist that the point $\sigma_{i}(t)\left(R_{\imath}^{\prime}\right)$ move positively and with constant speed.

3. $\mu_{i}$. (The shrinking of the $i^{\text {th }}$ arc.) Let $\mu_{i}(t)$ be the map of $K_{i}$ into $D$ such that $\left\|\mu_{i}(t)(P)-\mu_{i}(t)\left(P^{\prime}\right)\right\|=(1-t)\left\|P-P^{\prime}\right\|$ for $P, P^{\prime} \in K_{i}, \mu_{i}(t)\left(R_{i}\right)=R_{i}$ for all $t \leqq 1$, and for any $\rho<1, \mu_{i}(\rho t), 0 \leqq$ $t \leqq 1$ defines a parallel isotopy of the inclusion map $K_{i} \rightarrow D$. The map $\mu_{2}(t)$ will be useful mainly in defining other isotopies.

4. $\rho_{i j}, 1 \leqq i, j \leqq n, i \neq j$. (Pull the $i^{\text {th }}$ arc through the $j^{\text {th }}$ arc.) Let $\rho_{i j}(t)$ be the parallel isotopy of the inclusion map $K_{i} \rightarrow D$ such that $\rho_{i j}(t)=\mu_{2}(3 / 2 t), 0 \leqq t \leqq 1 / 3 ;\left\|\rho_{i j}(t)(P)-\rho_{i j}(t)(Q)\right\|=1 / 2\|P-Q\|$ for any $P, Q \in K_{i}, 1 / 3 \leqq t \leqq 2 / 3 ; \rho_{i j}(t)\left(R_{i}\right)$ moves positively with constant speed once around a circle in $P_{z y}$ whose diameter is $\left[R_{i}, R_{j}\right]$, $1 / 3 \leqq t \leqq 2 / 3 ;$ and $\rho_{i j}(t)=\mu_{i}(3 / 2-3 / 2 t), 2 / 3 \leqq t \leqq 1$.

5. $\omega_{i j}, 1 \leqq i \leqq n, 0 \leqq j \leqq n, j \neq i-1, i$. (Pull the $i^{\text {th }}$ arc between the $j^{\text {th }}$ and $j+1^{\text {st }}$ arcs or around the end arcs.) Let $\omega_{i j}(t)$ be the parallel isotopy of the inclusion map $K_{i} \rightarrow D$ such that $\omega_{i j}(t)=$ $\mu_{i}(3 / 2 t), 0 \leqq t \leqq 1 / 3 ;\left\|\omega_{i j}(t)(P)-\omega_{i j}(t)(Q)\right\|=1 / 2\|P-Q\|$ for any $P, Q \in K_{i}, 1 / 3 \leqq t \leqq 2 / 3 ; \omega_{i j}(t)\left(R_{i}\right)$ moves positively with constant speed once around a circle in $P_{z y}$ whose diameter is $\left[R_{\imath},(0,0,8 j+4)\right]$, $1 / 3 \leqq t \leqq 2 / 3 ;$ and $\omega_{i j}(t)=\mu(3 / 2-3 / 2 t), 2 / 3 \leqq t \leqq 1$.

We would like to associate with each of the previous isotopies an ambient isotopy of the pair $(D, A)$. To justify this we state the following theorem which is proved in [3], p. 154. 
THEOREM 1. (The $n$-isotopy extension theorem.) Let $F: M \times$ $I^{n} \rightarrow Q \times I^{n}, M$ and $Q P$. L. manifolds, $M$ compact, be an $n$-isotopy which is proper and locally unknotted. Then there exists an ambient $n$-isotopy $H$ of $Q$ with $H\left(F_{0} \times I^{n}\right)=F$.

Although it is not stated in [7], we may also assume that the ambient isotopy $H$ has compact support.

If $\varphi_{i}$ is one of the isotopies $\tau_{i}, \sigma_{i}, \rho_{i j}$ or $\omega_{i j}$, then we may restrict $\varphi_{i}$ to be an isotopy of an inclusion map $H_{i} \rightarrow D$ or $H_{i} \cup H_{i+1} \rightarrow D$. We consider $\varphi_{\imath}$ as an isotopy of the set $H_{i}$ (resp. $H_{i} \cup H_{i+1}$ ) in the manifold $(D-A) \cup a_{i}$ (resp. $\left.(D-A) \cup a_{i} \cup a_{i+1}\right)$. We now use the preceding theorem to obtain an ambient isotopic extension, which we shall also call $\varphi_{i}$, of the manifold $(D-A) \cup a_{i}$ (resp. $(D-A) \cup a_{\imath} \cup$ $\left.a_{i+1}\right)$. We can, by redefining if necessary, arrange that the ambient extension agree with the original isotopy on the set $K_{i}$ (or $K_{i} \cup K_{i+1}$ ). Since the ambient extension has compact support, we may extend it further to all of $D$. We shall also denote the extension of $\mathscr{P}_{i}$ to $D$ by $\varphi_{i}$, and the homeomorphism of $(D, A)$ it induces by the same Greek letter. (i.e. The isotopy $\rho_{i j}(t)$ extends to the ambient isotopy $\rho_{i j}(t)$ of $D$ which induces the homeomorphism $\rho_{i j}$ of $(D, A)$ equal to $\rho_{i j}(1)$.) We can easily arrange, and shall assume, that the homeomorphisms $\rho_{i j}$ and $\omega_{i j}$ are fixed in a neighborhood of $A$, that $\tau_{i}$ is fixed in a neighborhood of $A-a_{i}$ and is isometric in a neighborhood of $a_{i}$, that $\sigma_{i}$ is fixed in a neighborhood of $A-\left(a_{i} \cup a_{i+1}\right)$ and is isometric in a neighborhood of $a_{i} \cup a_{\imath+1}$, and that all the homeomorphisms $\rho_{i j}$, $\omega_{i j}, \tau_{i}$, and $\sigma_{i}$ have compact support. Moreover, by restricting to the set $(\partial D, A \cap \partial D)$, the homeomorphisms $\tau_{i}, \sigma_{i}, \rho_{\imath \jmath}$, ahd $\omega_{\imath j}$ define homeomorphisms of $(\partial D, A \cap \partial D)$ which we continue to denote by the same letter. The next lemma shows they are well defined as elements of $\mathscr{A}_{k}$.

LEMMA 2. Let $M$ be a compact manifold and let $\varphi: M \times I^{2} \rightarrow Q$ be a proper locally unknotted 2-isotopy such that $\varphi(m, s, 1)=\varphi(m, 1, t)$ for $0 \leqq s, t \leqq 1$ and all $m \in M$. Let $\alpha_{s}$ and $\beta_{t}$ be ambient isotopic extensions of $\varphi(\cdot, s, 0)$ and $\varphi(\cdot, 0, t)$ respectively. Then $\alpha_{1}$ is isotopic to $\beta_{1}$ via an isotopy of $Q$ that leaves the points of the set $\varphi(M \times$ $(1,1))$ fixed.

Proof. It suffices to show there is an ambient 2-isotopy of $Q, \psi: Q \times I^{2} \rightarrow Q$ such that $\psi$ extends $\varphi$, and

$$
\begin{aligned}
& \psi \mid Q \times(s, 0)=\alpha_{s} \\
& \psi \mid Q \times(0, t)=\beta_{t} .
\end{aligned}
$$


Then $\psi \mid Q \times(1, t)$ followed by $\psi \mid Q \times(1-s, 1)$ provides the required isotopy between $a$ and $\beta$.

Let $H(s, t)$ be any ambient 2-isotopic extension of $Q$. Let $F(s, t)$ be defined as follows:

$$
F(s, t)= \begin{cases}H^{-1}(s-t, 0) \circ \alpha_{s-t} & \text { if } s \geqq t \\ H^{-1}(0, t-s) \circ \beta_{t-s} & \text { if } t \geqq s\end{cases}
$$

Then $F(s, t)$ extends the constant isotopy of $M$ in $Q$. Also $F \mid Q \times$ $(s, 0)=H^{-1} \circ \alpha_{s}$ and $F \mid Q \times(0, t)=H^{-1} \circ \beta_{t}$. Now $\psi=H \circ F$ is the desired extension.

It follows easily from Lemma 2 that the homeomorphisms $\left\{\sigma_{i}\right.$, $\left.\tau_{i}, \rho_{i j}, \omega_{i j}\right\}$ are well defined as elements of $\mathscr{A}_{n}$. To see that $\sigma_{i}$ is well defined, for example, let $\varphi(m, s, t)=\sigma_{i}(m, \max (s, t)), \alpha_{s}=\sigma_{i}^{\prime}(s)$, and $\beta_{t}=\sigma_{i}^{\prime \prime}(t)$ for $\sigma_{i}^{\prime}$ and $\sigma_{i}^{\prime \prime}$ two different ambient isotopic extensions of $\sigma_{i}$ and apply Lemma 2 .

3. The main theorem. Let $\mathscr{G}$ be the group of homeomorphisms consisting of all finite products of homeomorphisms on the following list and their inverses:

1. $\tau_{i}^{2}, 1 \leqq i \leqq n$.

2. $\rho_{i j}, 1 \leqq i, j \leqq n$.

3. $\omega_{i j}, 1 \leqq i \leqq n$.

4. $\xi_{i j}, 1 \leqq i \leqq j \leqq n$, where $\xi_{i j}=\left(\sigma_{i} \sigma_{i+1} \cdots \sigma_{j-1}\right) \sigma_{j}^{2}\left(\sigma_{i} \cdots \sigma_{j-1}\right)^{-1}$.

5. $\alpha(1)$ where $\alpha(t)$ is an $A$-isotopy. Any isotopy $\alpha(t)$ of $D$ that is fixed on $A$ and has support disjoint from $\{\infty\}$ will be called an $A$-isotopy. The homeomorphism $\alpha(1)$ will also be called an $A$-isotopy.

Theorem 3. If $\varphi$ is a homeomorphism of $D$ fixed on a neighborhood of $A$ with support contained in a ball centered at $(0,0,0)$, then $\varphi \in \mathscr{G}$.

Proof. Suppose $\varphi$ fixes the neighborhood $N_{1}$ of $A$. We may assume (by multiplying $\varphi$ by an $A$-isotopy, if need be) that $\varphi\left(F-N_{1}\right)$ is in general position with respect to $E$. We wish to arrange that $\varphi\left(B-N_{1}\right) \cap C=\varnothing$. We shall do this by decreasing the number of points in this intersection step by step. If the set $\left(F-N_{1}\right) \cap \varphi^{-1}(E)$ is not empty, then it is a compact 1-manifold. Suppose $\varphi\left(B-N_{1}\right) \cap$ $C \neq \varnothing$. Then for some $i,\left(F_{i}-N_{1}\right) \cap \varphi_{1}^{-1}(E)$ has a component that is an arc, $a$, (not a closed curve). The endpoints of this arc, $\left\{M_{1}\right.$, $\left.M_{2}\right\}$, define a closed subarc $\left[M_{1}, M_{2}\right]$ of $b_{i}$. We may assume that $\varphi\left(\left[M_{1}, M_{2}\right]\right) \cap E=\left\{\varphi\left(M_{1}\right), \varphi\left(M_{2}\right)\right\}$. (If not, we could find another arc with this property in the disc bounded by a and $\left[M_{1}, M_{2}\right]$.) At this point we refer the reader to Figure 2. The points $\varphi\left(M_{1}\right)$ and $\varphi\left(M_{2}\right)$ 


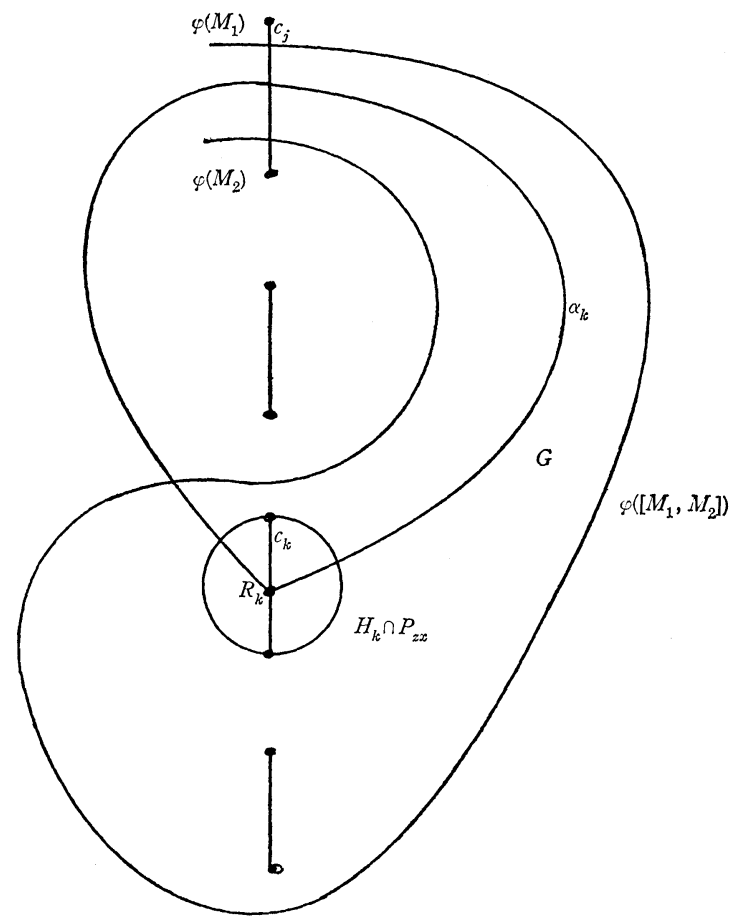

Figure 2

define a closed subarc $\left[\varphi\left(M_{1}\right), \varphi\left(M_{2}\right)\right]$ of $c_{j}$ for some $j$. We may assume (by composing with an $A$-isotopy fixed on $N_{1}$ not changing the number of points in $\varphi\left(B-N_{1}\right) \cap C$, if necessary) that $R_{j}$ belongs to the interior of this arc. If the disc $G$ whose boundary is $\left[\varphi\left(M_{1}\right)\right.$, $\left.\varphi\left(M_{2}\right)\right] \cup \varphi\left(\left[M_{1}, M_{2}\right]\right)$ contains none of the $\operatorname{arcs} c_{k}, k=1, \cdots, n, k \neq j$, it is easy to construct an $A$-isotopy $\psi$ supported on a neighborhood of $G$ such that $\varphi \psi\left(B-N_{1}\right) \cap C$ has two fewer points than $\varphi(B-$ $\left.N_{1}\right) \cup C$.

Suppose now $c_{k} \subset G$. We may assume (by composing with an $A$-isotopy fixed on $C$ and supported on a small neighborhood of $K_{k} \cap P_{z y}$, if necessary) that $K_{k} \cap P_{z y}$ belongs to the interior of $G$. Let $\alpha_{k}(t), 1 / 3 \leqq t \leqq 2 / 3$ be a parameterized simple closed curve beginning and ending at $R_{k}$ such that $\alpha_{k}(t) \cap C=\varnothing$ for all $t \neq 1 / 3$. $1 / 2,2 / 3$, such that $\alpha_{k}(1 / 2)$ lies in the interior of $\left[\varphi\left(M_{1}\right), \varphi\left(M_{2}\right)\right]$ and such that $\alpha_{k}(t)$ intersects $\partial G$ only twice, the second time when $t=$ $7 / 12$. Let $\varphi(t, \varepsilon)$ be an isotopy of $K_{k}$ defined by the following conditions: $\psi(t, \varepsilon)$ is a parallel isotopy, $\psi(t, \varepsilon)=\mu_{k}((3-\varepsilon) t), 0 \leqq t \leqq 1 / 3$, $\psi(t, \varepsilon)\left(K_{k}\right)$ is a half ball of radius $2 / 3 \varepsilon$ centered at $\alpha_{k}(t), 1 / 3<t \leqq 2 / 3$, $\psi(t, \varepsilon)=\psi(1-t, \varepsilon), 2 / 3 \leqq t \leqq 1$. If $0<\varepsilon<1$, we may, by Theorem 
1 , extend $\psi(t, \varepsilon)$ to an ambient isotopy of $D$, called $\bar{\psi}(t, \varepsilon)$ supported on a neighborhood of $K_{k} \cup \alpha_{k}[1 / 3,2 / 3]$. By choosing $\varepsilon$ small enough and the neighborhood of $K_{k} \cup \alpha_{k}[1 / 3,2 / 3]$ small enough, we may assume that $\bar{\psi} \varphi(1, \varepsilon)\left(B-N_{1}\right) \cap\left(C-\left[\varphi\left(M_{1}\right), \varphi\left(M_{2}\right)\right]\right)=\varphi\left(B-N_{1}\right) \cap C-$ $\left[\varphi\left(M_{1}\right), \varphi\left(M_{2}\right)\right]$, although new intersections of $\bar{\psi} \varphi(1, \varepsilon)\left(B-N_{1}\right)$ with $\left[\varphi\left(M_{1}\right), \varphi\left(M_{2}\right)\right]$ may be introduced. We can also arrange that $\bar{\psi}(1, \varepsilon)$ leaves a neighborhood, $N_{2} \subset N_{1}$, of $A$ fixed. Let $G_{1}$ be the disc bounded by $\left.\left[\bar{\psi} \varphi(1, \varepsilon)\left(M_{1}\right)\right], \bar{\psi} \varphi(1, \varepsilon)\left(M_{2}\right)\right]$ (which is the same as $\left[\varphi\left(M_{1}\right)\right.$, $\left.\left.\varphi\left(M_{2}\right)\right]\right)$ and $\bar{\psi} \varphi(1, \varepsilon)\left(\left[M_{1}, M_{2}\right]\right)$. We see that $c_{k}$ is not contained in $G_{1}$, but that $c_{p}$ is contained in $G_{1}$ if and only if it is contained in $G$. Next we must show $\bar{\psi}(1, \varepsilon)$ is in $\mathscr{G}$.

The curve $\bar{\psi}(t, \varepsilon)\left(R_{k}\right)$ defines an element of the grous $\pi_{1}\left(\left(P_{z y}-\right.\right.$ $\left.C) \cup c_{k} \cup\left(c_{j}-\left\{R_{j}^{\prime}, R_{j}^{\prime \prime}\right\}\right)\right)$. This group is a free group generated by the closed curves $\omega_{k j}(t)\left(R_{k}\right), 0 \leqq j \leqq n, j \neq k-1, k$ and $\rho_{k j}(t)\left(R_{k}\right)$, and so $\bar{\psi}(t, \varepsilon)\left(R_{k}\right)$ is homotopic to a product of these curves, $\beta(t)$. Thus there is a map $f: I^{2} \rightarrow\left(P_{z y}-C\right) \cup c_{k} \cup\left(c_{j}-\left\{R_{j}^{\prime}, R_{j}^{\prime \prime}\right\}\right)$ such that $f(0, t)=\bar{\psi}(t, \varepsilon)\left(R_{k}\right), f(s, 0)=\beta(s)$, and $f(1, t)=f(s, 1)=R_{k}$ for $0 \leqq s$, $t \leqq 1$. There is a continuous function $\eta(s, t)$ such that $\eta(0, t)=$ radius $\bar{\psi}(t, \varepsilon)\left(K_{k}\right), \eta(s, 0)=$ radius $\bar{\beta}(t)\left(K_{k}\right)$, where $\bar{\beta}(t)$ is the product of the isotopies in $\mathscr{L}$ that covers $\beta(t), \eta(s, 1)=\eta(1, t)=2$, and such that $\eta(s, t)$ is less than the distance from $f(s, t)$ to $A-a_{k}$. Let $F(s, t)$ be the parallel 2-isotopy such that $F(s, t)\left(K_{k}\right)$ is a half ball of radius $\eta(s, t)$ centered at $f(s, t)$. It now follows directly from Lemma 2 that $\bar{\psi}(1, \varepsilon) \varepsilon \mathscr{G}$.

Continuing in this fashion we can find a sequence of elements in $\mathscr{G}, \bar{\psi}_{1}, \cdots, \bar{\psi}_{r}$ such that each one fixes a neighborhood $N_{r} \subset N_{1}$, of $\left.A, \bar{\psi}_{r} \cdots \bar{\psi}_{1} \varphi\left(B-N_{r}\right) \cap C-\left[\varphi\left(M_{1}\right), \varphi\left(M_{2}\right)\right]\right)=\varphi\left(B-N_{r}\right) \cap\left(C-\left[\varphi\left(M_{1}\right)\right.\right.$, $\left.\varphi\left(M_{2}\right)\right]$ ), and the disc $G_{r}$ bounded by the arcs $\left[\varphi\left(M_{1}\right), \varphi\left(M_{2}\right)\right]$ (equal $\left.\left[\varphi \bar{\psi}_{1} \ldots \bar{\psi}_{k}\left(M_{1}\right), \varphi \bar{\psi}_{1} \ldots \bar{\psi}_{k}\left(M_{2}\right)\right]\right)$ and $\varphi \bar{\psi}_{1} \cdots \bar{\psi}_{k}\left[M_{1}, M_{2}\right]$ contains none of the $\operatorname{arcs} c_{k}$. Now we find one last $A$-isotopy, $\bar{\psi}_{r+1}$, supported on a small neighborhood of $G_{r}$ such that $G_{r} \cap C \cap \bar{\psi}_{r+1} \cdots \bar{\psi}_{1} \phi(B-$ $\left.N_{r}\right)=\varnothing$.

Thus, letting $\widetilde{\psi}_{1}=\bar{\psi}_{r+1} \ldots \bar{\psi}_{1}, \widetilde{\psi}_{1}$ is in $\mathscr{G}$, $\widetilde{\psi}_{1}$ fixes a neighborhood $N_{r}$ of $A$, and $\varphi\left(B-N_{r}\right) \cap C$ has two more points in it than $\widetilde{\psi}_{1} \varphi\left(B-N_{r}\right) \cap C$. By repeating this process several times we find an element $\psi$ in $\mathscr{G}$, that fixes a neighborhood $M$ of $A$, such that $\psi \varphi(B-M) \cap C=\varnothing$.

Next we would like to arrange that $\psi \varphi$ leave the set $C$ fixed. Let $H$ be the disc bounded by $c_{1}$ and $\varphi \psi\left(b_{1}\right)$. (See Figure 3.) Suppose $H \cap\left(C-c_{1}\right) \neq \varnothing$. That is, suppose some $c_{k}, k \neq 1$, is contained in the interior of $H$. By a procedure analogous to the process carried out in the last two paragraphs for removing the arcs $c_{k}$ from the $\operatorname{disc} G$, we may find an element $\theta_{1}$ fixed on a neighborhood $N$ of $A$ such that: $\theta_{1} \psi \varphi(B-N) \cap C=\varnothing, \theta_{1} \in \mathscr{G}$, and the disc $H_{1}$ bounded by 


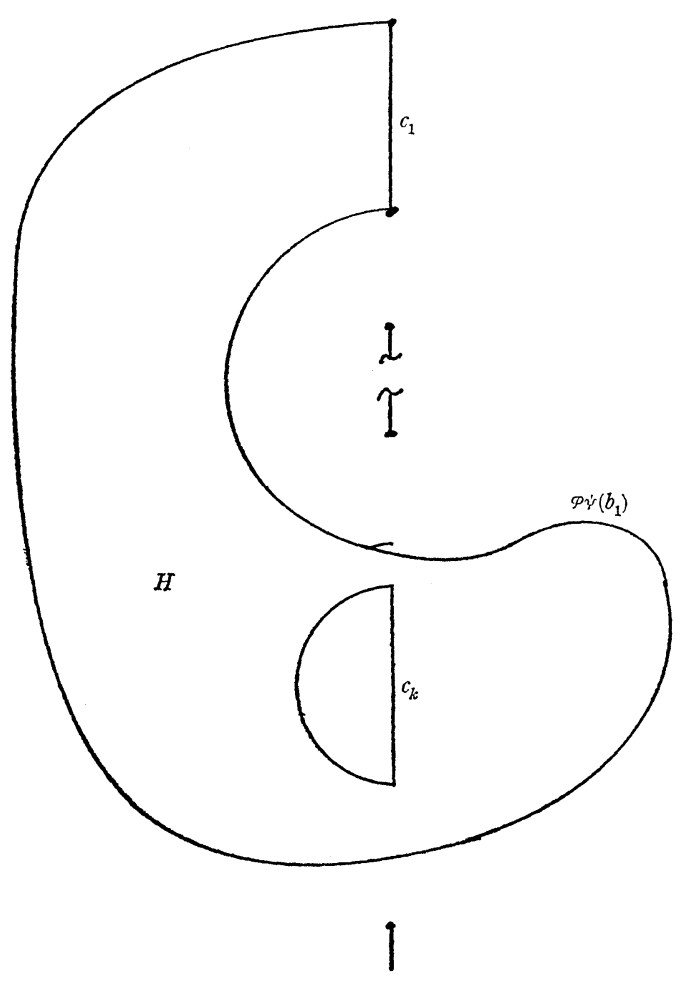

Figure 3

$\theta_{1} \psi \varphi\left(b_{1}\right)$ and $c_{1}$ satisfies $H_{1} \cap\left(C-c_{1}\right)=\varnothing$. Since $c_{1}$ is ambient $A$ isotopic to $b_{1}, \theta_{1} \psi \varphi\left(c_{1}\right)$ is ambient $A$-isotopic to $\theta_{1} \psi \varphi\left(b_{1}\right)$ which is in turn ambient $A$-isotopic to $c_{1} \cdot\left(\theta_{1} \psi \varphi\left(b_{1}\right)\right.$ and $c_{1}$ bound the disc $\left.H_{1}.\right)$ Thus there is an $A$-isotopy $\theta_{1}^{\prime}$ such that $\theta_{1}^{\prime} \theta_{1} \psi \varphi$ fixes each point of $c_{1}$. Continuing in this manner we find elements of $\mathscr{G}, \theta_{2}, \cdots, \theta_{n}$, and $\theta_{2}^{\prime}, \cdots, \theta_{n}^{\prime}$ such that $\theta_{n}^{\prime} \theta_{n} \cdots \theta_{1}^{\prime} \theta_{1} \psi \varphi$ fixes every point of $C$. (Note that by their construction, $\theta_{k}$ and $\theta_{k}^{\prime}$ fix the points of $c_{j}$ for $j<k$.) If we let $\psi_{1}=\theta_{n}^{\prime} \theta_{n} \cdots \theta_{1}^{\prime} \theta_{1} \psi$, then $\psi_{1} \in \mathscr{G}$ and $\psi_{1} \varphi$ fixes the points of C.

From this point to the end of the proof we shall denote the restriction of a homeomorphism of $D$ to $\partial D$ by placing a bar under it. $\quad \psi_{1} \rho$ defines an element of the subgroup generated by orientation preserving homeomorphisms of the mapping class group of $P_{z y}-\left\{R_{1}\right.$, $\left.\cdots, R_{n}\right\}$. It is shown in [1] and [2] that this group is generated by $\xi_{i j}, 1 \leqq i, j \leqq n-1$. Thus there is a homeomorphism $\psi_{2}$ of $D$ that is a product of $\xi_{i j}, 1 \leqq i, j \leqq n-1$, and $\psi_{2} \psi_{1} \varphi$ is isotopic to the identity via an isotopy fixing $R_{1}, \cdots, R_{n}$. Thus also $\psi_{2} \psi_{1} \varphi$ is isotopic to the identity via an isotopy fixing $R_{1}, \cdots, R_{n}$ (first isotopy on the boundary, then use the Alexander isotopy inside). Let $\theta_{t}$ be such an isotopy. Let $K(\varepsilon, k)$ be the hemisphere of radius $\varepsilon$ centered at $R_{k}$. 
If $\varepsilon>0$ is small enough then $\theta_{t}(K(\varepsilon, k)) \cap H_{k}=\varnothing$ for $k=1, \cdots, n$, $0 \leqq t \leqq 1$. Let $\rho(s)=\mu_{k}^{\prime}((1-\varepsilon / 2) s) \mu_{k-1}^{\prime}((1-\varepsilon / 2) s) \cdots \mu_{1}^{\prime}((1-\varepsilon / 2) s)$, where $\mu_{i}^{\prime}(t)$ is an ambient isotopic extension of $\mu_{i}$ supported on a small neighborhood of $K_{i}$. Thus $\rho$ pushes each half ball $K_{k}$ inside $K(\varepsilon, k)$. The expression $\underline{\rho}(s) \underline{\theta \rho}(s)$ defines an isotopy between $\underline{\theta}$ and $\rho^{-1} \theta \rho$ in the mapping class group of $P_{z y}-\left\{R_{1}^{\prime}, R_{1}^{\prime \prime}, R_{2}^{\prime}, R_{2}^{\prime \prime}, \cdots, R_{n}^{\prime \prime}\right\}$. We note that under the isotopy $\rho^{-1} \theta_{t} \rho$ the paths of $R_{k}^{\prime}$ and $R_{k}^{\prime \prime}$ never leave $K_{k} \cap P_{z y}$. The mapping class group of $U\left(K_{k} \cap P_{z y}\right)-\left\{R_{k}^{\prime}, R_{k}^{\prime \prime}\right\}$ is generated by $\tau_{k}^{2}$ where $U\left(K_{k} \cap P_{z y}\right)$ is a small neighborhood of $K_{k} \cap P_{z y}$. Thus there are integers $p_{1}, \cdots, p_{n}$ such that $\underline{\tau}_{n}^{2 p_{n}} \ldots$ $\tau^{2 p_{1}}\left[\rho^{-1} \theta \rho \theta^{-1}\right] \theta$ is isotopic to the identity via an isotopy fixing $\left\{R_{1}^{\prime}, \cdots\right.$, $\left.R_{k}^{\prime \prime}\right\}$. Also $\theta$ is $A$-isotopic to $\rho \theta \rho^{-1}$. To see this, let

$$
F(s, t)=\rho(s)^{-1} \theta_{\max (s, t)} \rho(s)
$$

restricted to $A$ and apply Lemma 2. Thus there is a homeomorphism $\psi_{3} \in \mathscr{G}$ such that $\psi_{3} \psi_{2} \psi_{1} \phi$ is isotopic to the identity via an isotopy $\psi_{4}$ fixing $A \cap P_{z y}$. Let $\psi_{5}=\psi_{4} \psi_{3} \psi_{2} \psi_{1}$. Then $\psi_{5} \in \mathscr{G}$ and $\psi_{5} \rho$ fixes $A \cup \partial D$. The last step is to show $\psi_{5} \rho$ is $A$-isotopic to 1 . Let $\psi_{6}$ be an isotopy such that $\psi_{6}$ restricted to $\partial D \cup A$ is the identity, $\psi_{6} \psi_{5} \varphi(E) \cap E$ is a 1-manifold without boundary, and the number of components of $\psi_{6} \psi_{5} \varphi(E) \cap E$ is as small as possible. Then $\psi_{6} \psi_{5} \varphi(E) \cap$ $E=\partial E$ since if $C_{1}$ were a circle in $E \cup\left(\psi_{6} \psi_{5} \varphi\right)^{-1}(E)$ not containing any other circles, then $\varphi\left(C_{1}\right)$ would bound a disc in the interior of $E$ and also a disc in the interior of $\psi_{6} \psi_{5} \varphi(E)$, these two discs would form a sphere bounding a ball and we could isotopy away the intersection. Since $E_{k} \cup \psi_{6} \psi_{5} \varphi\left(E_{k}\right)$ is a sphere for each $k$, we can find another isotopy $\psi_{7}$ fixed on $A \cup \partial D$ such that $\psi_{7} \psi_{6} \psi_{5} \rho$ is the identity on $\partial D \cup E$.

We now need only one more isotopy, $\psi_{8}$, an Alexander isotopy fixed on $\partial D \cup E$ such that $\psi_{8} \psi_{7} \psi_{6} \psi_{5} \varphi=$ identity. The homeomorphism $\varphi$ equals $\psi_{5}^{-1} \psi_{6}^{-1} \psi_{7}^{-1} \psi_{8}^{-1}$, which belongs to $\mathscr{G}$ and we are done.

4. Generators for $\mathscr{A}_{k}$ and $\mathscr{F}_{k}$. Let $\mathscr{A}_{k}$ be the group of orientation preserving homeomorphisms of the pair $(D, A)$ modulo the subgroup of those homeomorphisms isotopic to the identity via an isotopy leaving the set $A$ invariant. Let $\mathscr{F}_{k}$ be the subgroup of $\mathscr{A}_{k}$ generated by homeomorphisms leaving the set $A$ fixed pointwise.

THEOREM 4. The group $\mathscr{F}_{k}$ is generated by the following set: $\left\{\tau_{2}^{2}, 1 \leqq i \leqq n ; \rho_{i j}, 1 \leqq i, j \leqq n ; \omega_{i j}, 1 \leqq i, j \leqq n, i \neq j, j-1 ; \xi_{i j}, 1 \leqq\right.$ $i \leqq j \leqq n\}$.

Proof. If $\phi \in \mathscr{F}_{k}$, we can easily find an $A$-isotopy $\psi$ such that 
$\psi \varphi$ fixes a neighborhood of $\{\infty\}$ and $\psi \varphi$ fixes a neighborhood of $A$. The theorem now follows immediately from Theorem 3.

THEOREM 5. The group $\mathscr{A}_{k}$ is generated by the following set: $\left\{\tau_{i}, 1 \leqq i \leqq n ; \rho_{i j}, 1 \leqq i, j \leqq n ; \omega_{i j}, 1 \leqq i, j \leqq n, i \neq j, j-1 ; \sigma_{i}, 1 \leqq\right.$ $i \leqq n-1\}$.

Proof. Let $\varphi$ belong to $\mathscr{A}_{k}$. The homeomorphism $\varphi$ permutes the components of $A$ and $\sigma_{i}, 1 \leqq i \leqq n-1$, generates the set of permutations of the components of $A$. Thus there is an element $\psi_{1}$ which is a product of the $\sigma_{i}, 1 \leqq i \leqq n-1$, such that $\psi_{1} \phi$ leaves each component of $A$ fixed as a set. The homeomorphism $\psi_{1} \varphi$ may reverse the orientation of various components of $A$. Since the homeomorphism $\tau_{i}$ reverses the orientation of the $i^{\text {th }}$ component of $A$ while leaving the other component fixed, there is a homeomorphism $\psi_{2}$ that is a product of $\tau_{i}, 1 \leqq i \leqq n$, such that $\psi_{2} \psi_{1} \rho$ leaves each component of $A$ fixed and preserves its orientation. Since each component of $A$ is an arc, it is easy to see that there is an isotopy $\psi_{3}$ of $D$, leaving the set $A$ fixed as a set and supported on a neighborhood of $A$ such that $\psi_{3} \psi_{2} \psi_{1} \phi$ leaves the set $A$ fixed pointwise. The proof of Theorem 5 now follows immediately from Theorem 4 and the observation that each $\xi_{i j}$ is a product of $\sigma_{i}, 1 \leqq i \leqq n-1$.

It is perhaps worthwhile to remak that the sets of generators in Theorems 4 and 5 can be made much smaller by utilizing the relations in the mapping class group. For example

$$
\tau_{i}=\left(\sigma_{i-1} \sigma_{1-2} \cdots \sigma_{1}\right) \tau_{1}\left(\sigma_{i-1} \cdots \sigma_{1}\right)^{-1}
$$

so $\tau_{2}, \cdots, \tau_{n}$ are unnecessary in Theorem 5 .

5. Concluding remarks. In [4] D. M. Dahm finds generators for the group of motions of $n$ unlinked, unknotted circles $C_{n}$ in $E^{3}$. This group is quite close to the group of orientation preserving homeomorphisms of $\left(E^{3}, C_{n}\right)$ modulo those isotopic to the identity via an isotopy fixing $C_{n}$ as a set. There are three types of generators:

1. flip the $i^{\text {th }}$ circle;

2. pull the $i^{\text {th }}$ circle through the $j^{\text {th }}$ circle;

3. exchange the $i^{\text {th }}$ and $j^{\text {th }}$ circles.

It can be seen that these are analogous to the generators of $\mathscr{A}_{k}$ in Theorem 5. Dahm proves that these elements generate the motion group by defining an isomorphism from the motion group to a subgroup of Aut $\left(\pi_{1}\left(E^{3}-C_{n}\right)\right) . \quad\left(\pi_{1}\left(E^{3}-C_{n}\right)\right.$ is free on $n$ generators). This method doesn't generalize to a proof of Theorem 5 as nontrivial elements of $\mathscr{A}_{k}$ can be constructed that induce the identity automorphism on $\pi_{1}(D-A)$. 
In [6] Deborah L. Goldsmith proves several theorems about the group of motions of a link in $S^{3}$. She constructs certain examples of motions of a link. Theorem 5 can be used to construct examples of motions of links in the following way: Let $\ell$ be a $k$-bridge link, let $A$ be defined as in $\S 2$ with $k$ arcs, let $\left(D^{\prime}, A^{\prime}\right)$ be another copy of $(D, A)$, and let $i$ be the map that identifies $D$ with $D^{\prime}$ restricted to $\partial D$. Then $\left(S^{3}, \ell\right)=(D, A) \cup_{\alpha}\left(D^{\prime}, A^{\prime}\right)$ for some $\alpha \in \mathscr{B}_{2 k}$, the $2 k$ string braid group of the sphere. Let $\mathscr{N}$ be the group $A_{k} \cap \alpha \cdot \mathscr{A}_{k} \alpha^{-1}$. Each element of $m$ of $\mathscr{N}$ induces a homeomorphism $\theta_{m}$ of $\left(S^{3}, \ell\right)$ and therefore a motion of the link $\ell$ by the following formula: $\theta_{m}(x)=\bar{m}(x)$ if $x \in D, \theta_{m}(x)=\overline{i^{-1} \alpha^{-1} m \alpha i(x)}$ if $x \in D^{\prime}$, where the bar indicates the homeomorphism is an extension from $(\partial D, \partial D \cap A)$ (or $\left.\left(\partial D^{\prime}, \partial D^{\prime} \cap A^{\prime}\right)\right)$ to $(D, A)$ (or $\left.\left(D^{\prime}, A^{\prime}\right)\right)$.

A plat presentation of a link $\ell,\left(S^{3}, \ell\right)=(D, A) \mathrm{U}_{\alpha}\left(D^{\prime}, A^{\prime}\right)$, can be thought of as a Heegaard splitting of the link. Equivalence classes of Heegaard splittings of links then correspond in a natural way to double cosets of $\mathscr{A}_{k}$ in $\mathscr{A}_{2 k}$.

In [3], it is shown that there is a natural connection between Heegaard splittings of 3 -manifolds and Heegaard splittings of knots and links. In particular (Theorem 8 of [3]) there is a one-to-one correspondence beetween equivalence classes of Heegaard splittings of genus two closed orientable 3 -manifolds and double cosets of $\mathscr{A}_{3}$ in $\mathscr{H}_{6}$.

The writer would like to express his gratitude to the referee for suggesting the proof of Lemma 2, which is considerably shorter than the original.

\section{REFERENCES}

1. J. S. Birman, Mapping class groups and their relationship to braid groups, Communications on Pure and Applied Mathematics, 22 (1969), 213-238.

$2 . \quad$ On braid groups, Communications on Pure and Applied Mathematics, 22 (1969), 41-72.

3. J. S. Birman and H. M. Hilden, Heegaard splittings of branched coverings of $S^{3}$, to appear in Trans. Amer. Math. Soc.

4. D. Dahm, A Generalization of Braid Theory, Ph.D. Thesis, Princeton, 1962.

5. F. A. Garside Thesis, Corpus Christi College, Oxford, 1965.

6. D. L. Goldsmith, Motions of Links in the 3-Sphere, to appear.

7. J. F. P. Hudson, Piecewise Linear Topology, W. A. Benjamin, Inc., New York, 1969.

Received August 26, 1974. This research was supported by NSF grant GP-34059.

UNIVERSITY OF HAWAII 


\title{
PACIFIC JOURNAL OF MATHEMATICS
}

\section{EDITORS}

RichaRd ARENS (Managing Editor)

University of California

Los Angeles, California 90024

\section{R. A. Beaumont}

University of Washington

Seattle, Washington 98105
J. DUGUNDJI

Department of Mathematics University of Southern California Los Angeles, California 90007

D. Gilbarg and J. Milgram

Stanford University

Stanford, California 94305

\section{ASSOCIATE EDITORS}
E. F. BECKENBACH
B. H. NeUmanN
F. WOLF
K. YoshIDA

\section{SUPPORTING INSTITUTIONS}

\author{
UNIVERSITY OF BRITISH COLUMBIA \\ CALIFORNIA INSTITUTE OF TECHNOLOGY \\ UNIVERSITY OF CALIFORNIA \\ MONTANA STATE UNIVERSITY \\ UNIVERSITY OF NEVADA \\ NEW MEXICO STATE UNIVERSITY \\ OREGON STATE UNIVERSITY \\ UNIVERSITY OF OREGON \\ OSAKA UNIVERSITY
}

\author{
UNIVERSITY OF SOUTHERN CALIFORNIA \\ STANFORD UNIVERSITY \\ UNIVERSITY OF TOKYO \\ UNIVERSITY OF UTAH \\ WASHINGTON STATE UNIVERSITY \\ UNIVERSITY OF WASHINGTON \\ AMERICAN MATHEMATICAL SOCIETY
}

The Supporting Institutions listed above contribute to the cost of publication of this Journal, but they are not owners or publishers and have no responsibility for its content or policies.

Mathematical papers intended for publication in the Pacific Journal of Mathematics should be in typed form or offset-reproduced, (not dittoed), double spaced with large margins. Underline Greek letters in red, German in green, and script in blue. The first paragraph or two must be capable of being used separately as a synopsis of the entire paper. Items of the bibliography should not be cited there unless absolutely necessary, in which case they must be identified by author and Journal, rather than by item number. Manuscripts, in triplicate, may be sent to any one of the editors. Please classify according to the scheme of Math. Reviews, Index to Vol. 39. All other communications should be addressed to the managing editor, or Elaine Barth, University of California, Los Angeles, California, 90024.

The Pacific Journal of Mathematics expects the author's institution to pay page charges, and reserves the right to delay publication for nonpayment of charges in case of financial emergency.

100 reprints are provided free for each article, only if page charges have been substantially paid. Additional copies may be obtained at cost in multiples of 50 .

The Pacific Journal of Mathematics is issued monthly as of January 1966. Regular subscription rate: $\$ 72.00$ a year (6 Vols., 12 issues). Special rate: $\$ 36.00$ a year to individual members of supporting institutions.

Subscriptions, orders for back numbers, and changes of address should be sent to Pacific Journal of Mathematics, 103 Highland Boulevard, Berkeley, California, 94708.

PUBLISHED BY PACIFIC JOURNAL OF MATHEMATICS, A NON-PROFIT CORPORATION

Printed at Kokusai Bunken Insatsusha (International Academic Printing Co., Ltd.), 8-8, 3-chome, Takadanobaba, Shinjuku-ku, Tokyo 160, Japan.

Copyright (C) 1975 by Pacific Journal of Mathematics Manufactured and first issued in Japan 


\section{Pacific Journal of Mathematics}

\section{Vol. 59, No. $2 \quad$ June, 1975}

Aharon Atzmon, A moment problem for positive measures on the unit disc ........

Peter W. Bates and Grant Bernard Gustafson, Green's function inequalities for

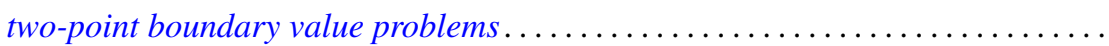

Howard Edwin Bell, Infinite subrings of infinite rings and near-rings ...........

Grahame Bennett, Victor Wayne Goodman and Charles Michael Newman, Norms of

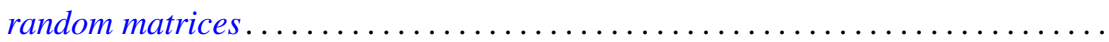

Beverly L. Brechner, Almost periodic homeomorphisms of $E^{2}$ are periodic.........

Beverly L. Brechner and R. Daniel Mauldin, Homeomorphisms of the plane ........

Jia-Arng Chao, Lusin area functions on local fields ......................

Frank Rimi DeMeyer, The Brauer group of polynomial rings ...............

M. V. Deshpande, Collectively compact sets and the ergodic theory of

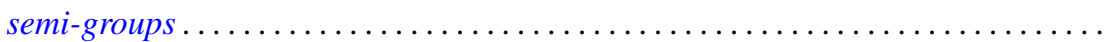

Raymond Frank Dickman and Jack Ray Porter, $\theta$-closed subsets of Hausdorff

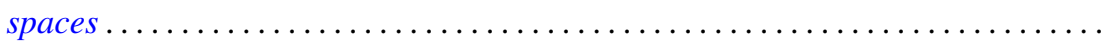

Charles P. Downey, Classification of singular integrals over a local field .......... 407

Daniel Reuven Farkas, Miscellany on Bieberbach group algebras . . . . . . . . . . . .

Peter A. Fowler, Infimum and domination principles in vector lattices . . . . . . . . . .

Barry J. Gardner, Some aspects of T-nilpotence. II: Lifting properties over

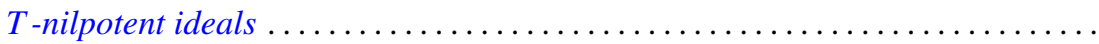

Gary Fred Gruenhage and Phillip Lee Zenor, Metrization of spaces with countable

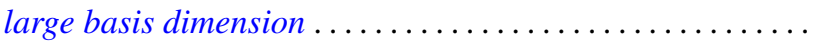

J. L. Hickman, Reducing series of ordinals...

Hugh M. Hilden, Generators for two groups related to the braid group ...

Tom (Roy Thomas Jr.) Jacob, Some matrix transformations on analytic sequence

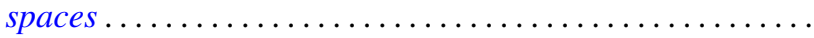

Elyahu Katz, Free products in the category of $k_{w}$-groups . .....

Tsang Hai Kuo, On conjugate Banach spaces with the Radon-Nikodým property...

Norman Eugene Liden, $K$-spaces, their antispaces and related mappings ...

Clinton M. Petty, Radon partitions in real linear spaces ........

Alan Saleski, A conditional entropy for the space of pseudo-Menger maps ....

Michael Singer, Elementary solutions of differential equations .

Eugene Spiegel and Allan Trojan, On semi-simple group algebras. I. . .

Charles Madison Stanton, Bounded analytic functions on a class of open Riemann

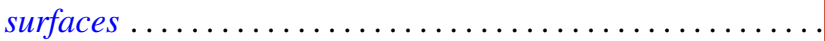

Sherman K. Stein, Transversals of Latin squares and their generalizations ....

Ivan Ernest Stux, Distribution of squarefree integers in non-linear sequences . . .

Lowell G. Sweet, On homogeneous algebras ................

Lowell G. Sweet, On doubly homogeneous algebras .......... .

Florian Vasilescu, The closed range modulus of operators ......

Arthur Anthony Yanushka, A characterization of the symplectic groups $\operatorname{PSp}(2 m, q)$

as rank 3 permutation groups... 